\title{
La necesidad de internacionalización: entrada de la Denominación de Origen Montilla-Moriles en los mercados BRIC
}

\author{
Mercedes LUQUE-VÍLCHEZ \\ Universidad de Burgos \\ mlvilches@ubu.es
}

Recibido: 25-06-2015

Aceptado: 29-10-2015

\begin{abstract}
RESUMEN
La competencia del sector vitivinícola españoles hoy más dura que nunca. Han surgido nuevos exportadores como Nueva Zelanda o Sudáfrica que ofrecen vinos de una calidad considerable y a unos precios bajos. Además otros factores como el contexto de crisis económica y el programa europeo de ayudas de arranque o los cambios de hábito de la población, han posicionado al sector vitivinícola español en un contexto en el que es imposible crear rentabilidad en el sector. Ante esta nueva situación, hay que destacar el papel de los países BRIC (Brasil, Rusia, India y China) como una oportunidad para el sector. Se trata de un conjunto de países cuyos índices micro y macroeconómicos se han desviado del conjunto, siendo así emergentes mercados potenciales. Es por ello que resulta muy atractivo determinar cuál podría ser el país más adecuado para introducir el vino español. Por ello, el objetivo de este trabajo es determinar la mejor manera de introducir el vino español, concretamente el vino con D.O Montilla-Moriles, en la zona BRIC. Para tal fin, esta investigación se basa en una metodología que comprende dos partes: una parte descriptiva del sector; y una segunda parte, basada en un análisis PEST (Bueno Campos, 1993). Debido a que la crisis económica ha sido uno de los factores clave que han forzado el sector vitivinícola para exportar el producto, este estudio abarca el período de 2007 a 2011 (momento más difícil de la crisis económica y financiera en España). Tras concluir el estudio, el mejor país para introducir el vino resulta ser Rusia.
\end{abstract}

Palabras clave: internacionalización, vino, crisis económica, BRIC. 


\title{
The need for internationalization: entry of the Denomination of Origin Montilla-Moriles in the BRIC markets
}

\begin{abstract}
Competition in the Spanish wine sector is today harder than ever. They are new exporters as New Zealand or South Africa, which offer wines with considerable quality wines and low price. Besides the economic crisis, and other factors such as the European aids program to pick vineyards have become Montilla-Moriles area in which it is impossible to create profitability, forcing her to have to reposition the product in foreign markets. Given this new situation, we should mention the role of the BRIC (Brazil, Russia, India and China) countries as an opportunity for the sector because they are emerging potential markets. Thus, the aim of this paper is to determine the best way to introduce the wine in the BRIC area. For that end, the paper is based on a methodology with two parts: a descriptive part supported by a statistics analysis, which describe wine sector in quantitative terms; and a second part, based on a PEST analysis (Bueno Campos, 1993). Because the economic crisis has been one of the key factors that have forced the wine sector to export the product, this study covers the period from 2007 to 2011 (most difficult time of the economic and financial crisis in Spain). Finally, the best country to introduce the wine resulted to be Russia.
\end{abstract}

Keywords: internationalization, wine, economic crisis, BRIC.

Sumario: 1. Introducción 2. Marco teórico 3. Metodología 4. Denominación de Origen Montilla-Moriles 5. Análisis del sector vitivinícola 6. Análisis PEST para el conjunto de los BRIC 7. Conclusiones.

\section{INTRODUCCIÓN}

Nuestro país es uno de los grandes productores mundiales de vino. Pertenece al denominado Viejo Mundo del Vino, que comprende a regiones con una larga historia en la producción de este caldo que proceden de Europa y del Norte de África. España es el primero en el ranking por superficie plantada, el tercero por producción (debido a un rendimiento menor que el de Francia e Italia) y el segundo exportador mundial en términos de volumen, aunque el tercero también en términos de valor como apunta la Organización Internacional del Vino-OIV (2011). En definitiva, el sector del vino es de extraordinaria relevancia ya no sólo en términos económicos, sociales o ambientales; sino además porque es un símbolo de identidad de nuestro país de cara al exterior.

Si bien hace unas décadas el vino era casi un alimento más a incluir dentro de la dieta mediterránea, poco a poco ha perdiendo importancia, y han ido apareciendo otro tipo de bebidas que le han ido sustituyendo. Aun así, resulta de gran interés destacar que según el Observatorio Español Mercado del Vino-OEMV (2012) aumentó el consumo de vino en los hogares españoles en un 2,9\% en volumen, 
debido a su mayoría al aumento de vinos sin denominación de origen y el aumento de sangrías y tintos de verano. A pesar de ello la facturación descendió un 1,5\%, suponiendo esto un descenso del precio importante.

Nuestra competencia tradicional había sido Italia y Francia, pero hoy debido a la creciente apertura de nuevos mercados que permite la libre circulación desencadenada (León y Pla, 2004) la competencia se ha vuelto más fuerte. Han surgido nuevos exportadores de vino contra los que nos es muy difícil luchar, pues ofrecen unos vinos de una calidad considerable y a unos precios bajos. Esto es a lo que se denomina el Nuevo Mundo del Vino. Son países emergentes como Nueva Zelanda o Sudáfrica, que por su situación geográfica pueden producir un producto similar al nuestro y a un precio más competitivo.

Desde que en 2008 empezase a florecer la crisis económica mundial el concepto de internacionalización ha ido tomando aún más relevancia y la decisión de las empresas de vender sus productos o servicios fuera de sus países de origen, es hoy por hoy, una de los más importantes objetivos estratégicos empresariales. Por esta razón se ha estudiado un periodo (hasta 2011-12) que es representativo de los momentos más difíciles de la crisis económica y financiera; la cual en la actualidad parece que empieza a hacerse poco a poco más leve.

El contexto de crisis económica, el surgimiento de fuerte competencia en nuevos mercados y otros factores como el programa europeo de ayudas de arranque y los cambios de hábito de la población han posicionado a España un panorama en el que es imposible crear rentabilidad en el sector, obligándola a tener que recolocar el producto en el mercado exterior. Ante esta nueva situación en la que se encuentra el mercado del vino y concretamente sus exportaciones hay que destacar el papel destacado de los países BRIC (Brasil, Rusia, India y China). Se trata de un conjunto de países cuyos índices macro y macroeconómicos se han desviado del conjunto, siendo así emergentes mercados potenciales.

Como una salida a la delicada situación del sector vitivinícola en España surge la posibilidad de exportar a los denominados Nuevos Mercados BRIC .Éstos países son Brasil, Rusia, India y China, los cuáles engloban casi al 50\% de la población mundial. Esto unido al interés y curiosidad que representan para estos ciudadanos la cultura española, y en general la mediterránea; hace que nuestro producto les sea muy atractivo. Es por ello que resulta muy atractivo determinar, y este es el objetivo principal de este artículo, cuál podría ser el país más adecuado para introducir nuestro producto. Esto se determina en esta investigación discrepando entre diferentes aspectos positivos y negativos de cada país (método PEST, ver más adelante), lo cual a su vez ayuda a establecer el método de entrada en el país elegido. Tras realizar el estudio el país BRIC más adecuado para vender el vino con Denominación de Origen Montilla-Moriles resulta ser Rusia.

El resto del documento está estructurado de la siguiente manera. La segunda sección presenta un marco teórico para el estudio de los procesos de internacionalización. En la tercera sección se describe la metodología usada en la 
investigación, la cual se compone de dos partes principales: una parte descriptiva apoyada en estadística, que nos aporta la descripción general del panorama vitivinícola en términos cuantitativos; y una segunda parte, basada en un análisis PEST, que ofrece la posibilidad de entender de manera clara y sistemática, algunos de los aspectos que va a influir a la hora de introducirnos en un nuevo país. Este último se trata del estudio de cuatro bloques temáticos: factores político legales $(\mathrm{P})$; factores económicos (E); factores socio-culturales (S); y factores tecnológicos (T) (Bueno Campos, 1993). La cuarta sección se presenta algunas peculiaridades de la Denominación de Origen Montilla-Moriles. Las secciones quinta y sexta realizan un análisis comentado anteriormente. Por último, la sección séptima ofrece algunas conclusiones finales

\section{MARCO TEÓRICO}

El contexto económico, político y social se caracteriza hoy en día por un mayor nivel de complejidad, interdependencia y flexibilidad que ha propiciado que las relaciones entre distintos países se intensifiquen y como mejor exponente de este proceso se encuentra la internacionalización de las economías y de sus empresas (Chevalier 1995, Dunning 1994 y Ohmae 1991). Esto provoca una creciente interdependencia entre los países unificando mercados, sociedades y culturas, por medio de una serie de transformaciones económicas, sociales y políticas que le dan un carácter global. Es por esto que el fenómeno de la internacionalización ha adquirido una elevada importancia tanto en el ámbito empresarial (Abbby y Slater, 1998). Hablamos así de un único mercado mundial interrelacionado que ha ofrecido nuevas posibilidades a las empresas al encontrarse con un mercado más amplio y abierto, aunque también ha traído nuevas amenazas para éstas como un aumento de la competencia. Sin duda, sobrepasar las fronteras del país supone una oportunidad de crecimiento para las empresas.

Cuando una empresa quiere llevar a cabo un proceso de internacionalización puede perseguir una serie de objetivos (Grant, 1996; Porter, 1998; Navas y Guerras, 2002):

a) Búsqueda de eficiencia

El hecho de internacionalizar produce dos consecuencias relacionadas con la eficiencia:

a.1) Si salimos fuera, al estar presente en varios países, se puede alcanzar la economía de escala.

a.2) Se minimiza el riesgo al fracaso, al aumentar la diversificación.

b) Explorar nuevos mercados.

Esto está relacionado con la búsqueda de nuevos mercados y con el análisis de sus características. Lo que se pretende es encontrar mercados atractivos, que por su tasa de crecimiento o por su tamaño sean óptimos para la internacionalización. Este es el objetivo más relacionado con este estudio ya que los países en los que se 
centra son Brasil, Rusia, India y China (BRIC); que se han convertido en el objetivo de muchas empresas por su espectacular crecimiento.

\section{c) Obtención de recursos}

La obtención de recursos puede ser más difícil de obtener en unos países que en otros .Los recursos por sí solos no generan ventaja competitiva: deben trabajar de forma conjunta para crear una capacidad organizativa, la cual es la esencia de unos resultados superiores. Hay veces en las que parte de los recursos no se encuentran en el país de origen o se encuentran obsoletos y se deben buscar fuera.

Ejemplo: Muchas empresas escandinavas han abierto centros de distribución en Holanda, ya que ésta cuenta por ejemplo, con una gran eficiencia en sus puertos.

\section{METODOLOGÍA}

La metodología usada en este trabajo se basa en dos partes: una parte descriptiva apoyada en estadística (hasta años 2011-2012), que nos aporta la descripción general del panorama vitivinícola en términos cuantitativos; y una segunda parte, basada en un análisis PEST, que ofrece la posibilidad de entender de manera clara y sistemática, algunos de los aspectos que va a influir a la hora de introducirnos en un nuevo país. Este último se trata del estudio de cuatro bloques temáticos: factores político legales $(\mathrm{P})$; factores económicos $(\mathrm{E})$; factores socioculturales (S); y factores tecnológicos (T) (Bueno Campos, 1993).

El hecho de que nuestro estudio refleje datos hasta el periodo 2011-12, lo hace representativo del periodo de crisis, que como hemos mencionado ha sido uno de los factores que junto con otros (surgimiento del Nuevo Mundo del Vino, el programa europeo de ayudas de arranque, los cambios de hábito de la población) han desencadenado la continua pérdida de rentabilidad en el sector.

\section{DENOMINACIÓN DE ORIGEN MONTILLA-MORILES}

El concepto de denominación de origen se establece en nuestro país con el Estatuto del Vino de 1932, que reconoce legalmente la práctica comercial que relaciona la calidad específica de los vinos con su zona geográfica de procedencia. Esta vinculación es común a todas las culturas mediterráneas, siendo en los primeros años de este siglo cuando se empiezan a establecer normas legales con el fin de evitar competencias y fraudes al consumidor (Sáinz, 2001).

Los Vinos con Denominación de Origen (D.O.), ver en la tabla 1 del anexo, reciben el nombre de una zona delimitada, región, comarca o localidad que ha sido reconocida legalmente para englobar a aquellos caldos que contienen unas determinadas características similares derivadas por ejemplo de la zona geográfica en que está el cultivo.

Este tipo no sólo no sólo son famosos por unos valores naturales, sino también por el reconocimiento y prestigio que le es asociado. Son asociados a una forma de vida, a una cultura. España cuenta con 89 zonas de producción de vinos de calidad 
con Denominación de Origen Protegida (DOP), de ellas 67 son con Denominación de Origen, 2 con Denominación de Origen Calificada, 6 son Denominación de Vinos de Calidad con Indicación Geográfica, las cuales siguiendo el modelo europeo de producción, mantienen un estricto control sobre la cantidad producida, las prácticas enológicas, y la calidad de los vinos que se producen en cada zona. Las primeras Denominaciones autorizadas se aprobaron en 1932, y se trata de Jerez-Xères-Sherry, Manzanilla de Sanlúcar de Barrameda, Málaga, MontillaMoriles, Rioja, Tarragona, Priorato, Alella, Utiel Requena, Valencia, Alicante, Ribeiro, Cariñena, Penedés, Condado de Huelva, Valdepeñas, La Mancha, Navarra y Rueda.

Resulta importante destacar que existe una categoría superior a la de DOP. Es la DOPCa, Denominación Propia de Origen Protegida Certificada. Estos vinos comprenden una categoría que se encuentra reservada para aquellos que tiene gran tradición y alta calidad .están sometidos a altos controles y regulaciones .Entre los requisitos se exigen que haya pasado al menos diez años desde la DOP. Además de esto se debe cumplir que todos los productos se comercialicen embotellados desde bodegas situadas en la misma zona de producción y un estricto sistema de control en todo el proceso. La denominación de origen La Rioja es DOPCa.

\section{ANÁLISIS DEL SECTOR VITIVINÍCOLA}

La producción de vino en el mundo está presente en todo el mundo, pero existen grandes zonas productoras mientras que en otras no se alcanzan los niveles suficientes.

Italia, España y Francia conforman el llamado Antiguo Mundo del Vino y concentran la mitad de la producción (Rossano, 2001). Sin embargo, los diez primeros productores (estos tres más EEUU, Argentina, China, Australia, Sudáfrica, Alemania y Chile) suponen el $80 \%$ de la producción mundial de vino según OVI (2011).

La tendencia que hay a nivel mundial durante los últimos años es de reducción gradual de las superficies de viñedo (figura 1). Esto es debido a la disminución de la demanda a partir de los años 80 .

Según datos de la OIV (2011) la superficie vitícola mundial disminuyó en 94.000 hectáreas respecto a 2010, estimándose el total mundial en 7.495 .000 ha. El viñedo comunitario total (UE-27) está reduciendo progresivamente su superficie plantada, pasando de las 3.792.000 has en el año 2008 a las 3.530.000 has en el año 2011. 


\section{Figura 1}

Evolución de la superficie mundial de cultivo de vid en la última década
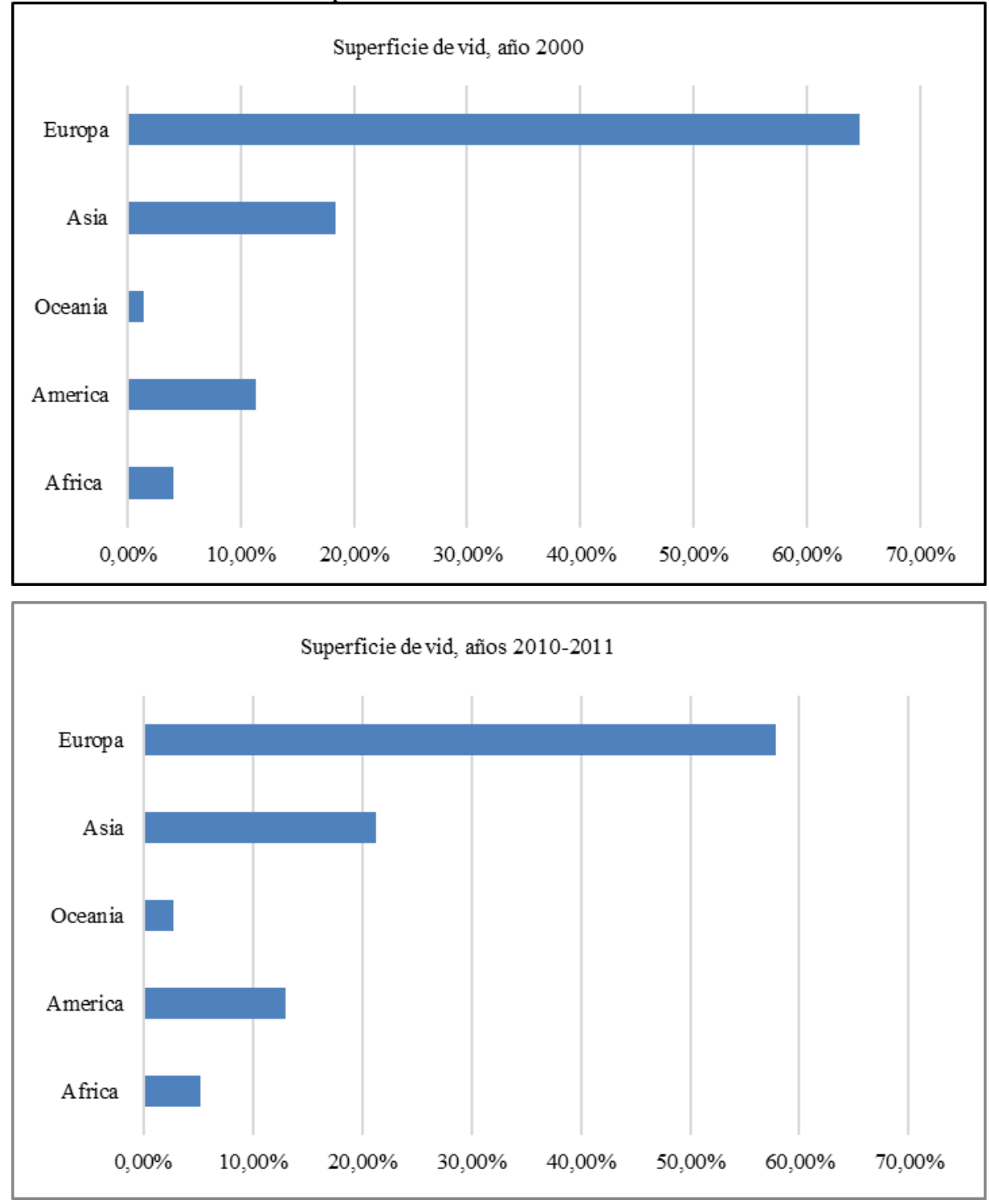

Fuente: Elaboración propia a partir de los Informes de la OIV (2010, 2011)

La disminución del viñedo comunitario queda compensada por el mantenimiento de las superficies plantadas del resto del mundo. Mientras disminuyen las plantaciones en Argentina y Turquía, éstas crecen en China y Australia y se mantienen casi invariables en EE.UU. y Sudáfrica. 
Lo más representativo es la pérdida constante de representatividad del continente europeo (figura 1). La superficie de vid del total mundial que representa Europa ha pasado de estar en torno al 65\% en el año 2000 a no alcanzar el $60 \%$ en el periodo 2010-11. El cultivo y producción de uva en este continente se esté estancando en comparación con el gran crecimiento que están experimentando los denominados Vinos del Nuevo Mundo.

Es importante destacar que si bien desde 1999 la superficie que supone el continente europeo se ha ido reduciendo, cuando más acusado ha sido este fenómeno ha sido a partir de 2008 con la implantación de la Organización Común del Mercado Europeo (en adelante OCM). Ésta ha supuesto un estímulo a que los agricultores arrancaran millones de hectáreas en muy poco tiempo. España ha sido el país más afectado por esta normativa. La reducción global de viñedo ha sido de un 5\% en 2009 respecto a 2008 (OIV, 2011). Muchos agricultores no sólo se han acogido a esta medida sino que el abandono total ha sido poco superior al debido a los incentivos (financiados con fondos comunitarios) lo que evidencia que hay una relación directa entre la nueva normativa, el abandono de cultivos y el retroceso de la superficie cultivada (OIV, 2009). Después de analizar la superficie cultivada de uva, es lógico pensar el descenso que ha sufrido la producción de uva a nivel mundial.

En la figura 2 se muestra que Europa, como ocurre con el tema de la superficie cultivada, sigue siendo la de mayor hegemonía también en cuanto a nivel de producción de uva como consecuencia de que este continente se sitúa los países del nombrado anteriormente Viejo Mundo del Vino.

Si contemplamos los datos de la tabla 1, observamos como dentro de Europa se produce más de la mitad del vino de todo el mundo. Por otro lado, se observa que la tendencia generalizada dentro del continente europeo es la del descenso de producción. Sin embargo los países pertenecientes al denominado Nuevo Mundo del Vino aumentan sus cifras de producción (Argentina, Chile). De continuar así, podrían pronto representar superioridad en la producción mundial de vino frente a los países de tradición vitivinícola como España, Francia e Italia.

A través de los datos de producción de vino y uva mundiales analizados se llega a la conclusión de que la viticultura se centra principalmente en Europa. Si bien la EU supone el 59\% de la producción mundial de vino (tabla 1), sólo produce el $44 \%$ del volumen total de uva (figura 2). La diferencia de ese $13 \%$ es debida a que países como Grecia o Italia son los principales productores de uvas de mesa y pasas; a diferencia de España donde se dedica la gran mayoría de la uva al sector vitivinícola.

Concretamente, según OVI (2011) la producción de vino de la Unión Europea en 2011 se sitúa en 156,9 millones de hl, ligeramente superior a la de $2010(156,4$ millones), aunque muy inferior a la de 2009, cuando se rozaban los 163 millones de hl (figura 3). A continuación se da una pincelada de cómo ha sido la evolución en la producción de cada uno de los países de la Europa de los 27. 


\section{Figura 2}

Reparto de la producción mundial de uva por continentes, 2010.

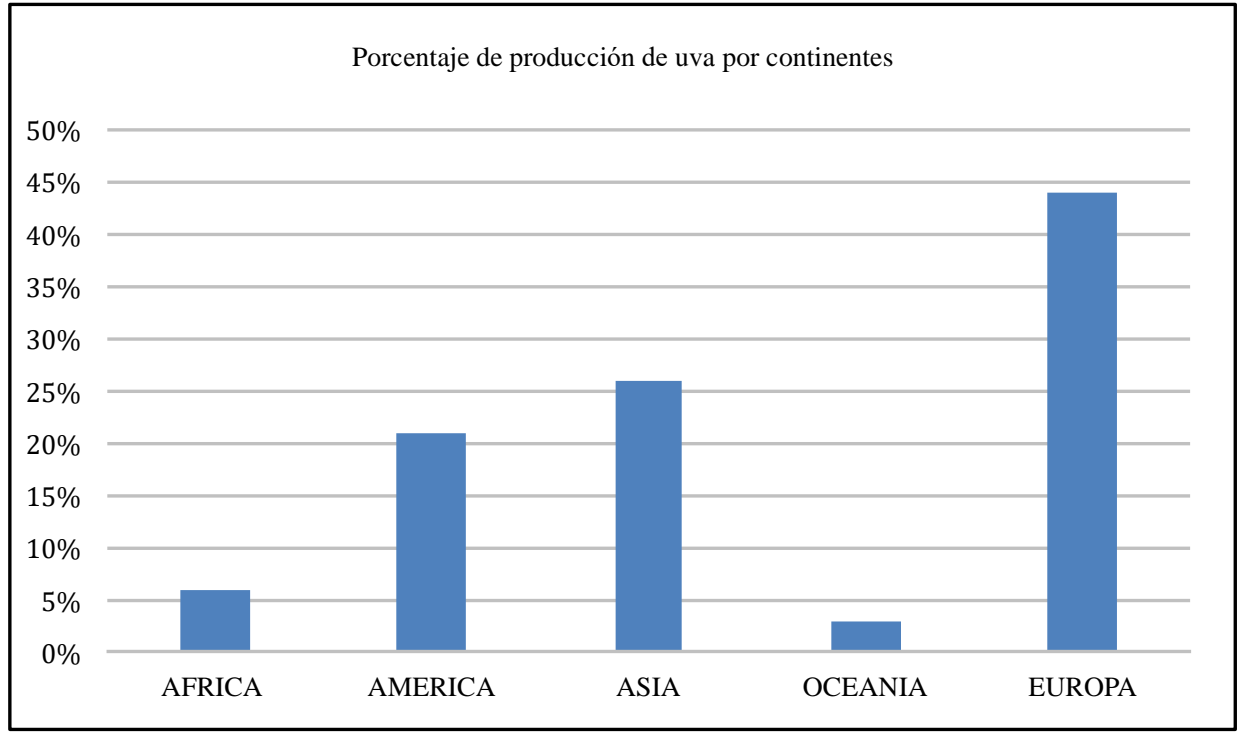

Fuente: elaboración propia a partir de los Informes de la OIV $(2010,2011)$

\section{Tabla 1}

Producción de vino mundial

\begin{tabular}{|lcccc|}
\hline MILES hl & $\mathbf{2 0 0 9}$ & $\mathbf{2 0 1 0}$ & $\mathbf{2 0 1 1}$ & \% S/TOTAL \\
\hline Italia & 47.450 & 48.525 & 49.633 & $18,70 \%$ \\
Francia & 46.361 & 45.704 & 41.580 & $15,65 \%$ \\
España & 35.166 & 35.235 & 34.300 & $12,90 \%$ \\
Otros UE & 33.921 & 26.912 & 31.371 & $11,80 \%$ \\
TOTAL UE & 33.921 & 156.376 & 156.884 & $59,00 \%$ \\
\hline EEUU & 21.690 & 20.887 & 18.740 & $7,10 \%$ \\
Argentina & 12.135 & 16.250 & 15.473 & $5,80 \%$ \\
Australia & 11.710 & 11.240 & 11.010 & $4,10 \%$ \\
Chile & 10.093 & 9.152 & 10.572 & $4,00 \%$ \\
TOTAL NO UE & 108.302 & 108.724 & 108.916 & $41,00 \%$ \\
\hline TOTAL & 271.200 & 265.100 & 265.000 & $100,00 \%$ \\
\hline
\end{tabular}

Fuente: OEMV a través de los datos de OIV (2009, 2010, 2011) 


\section{Figura 3}

Evolución de la producción de vino en la UE (en Miohl) global

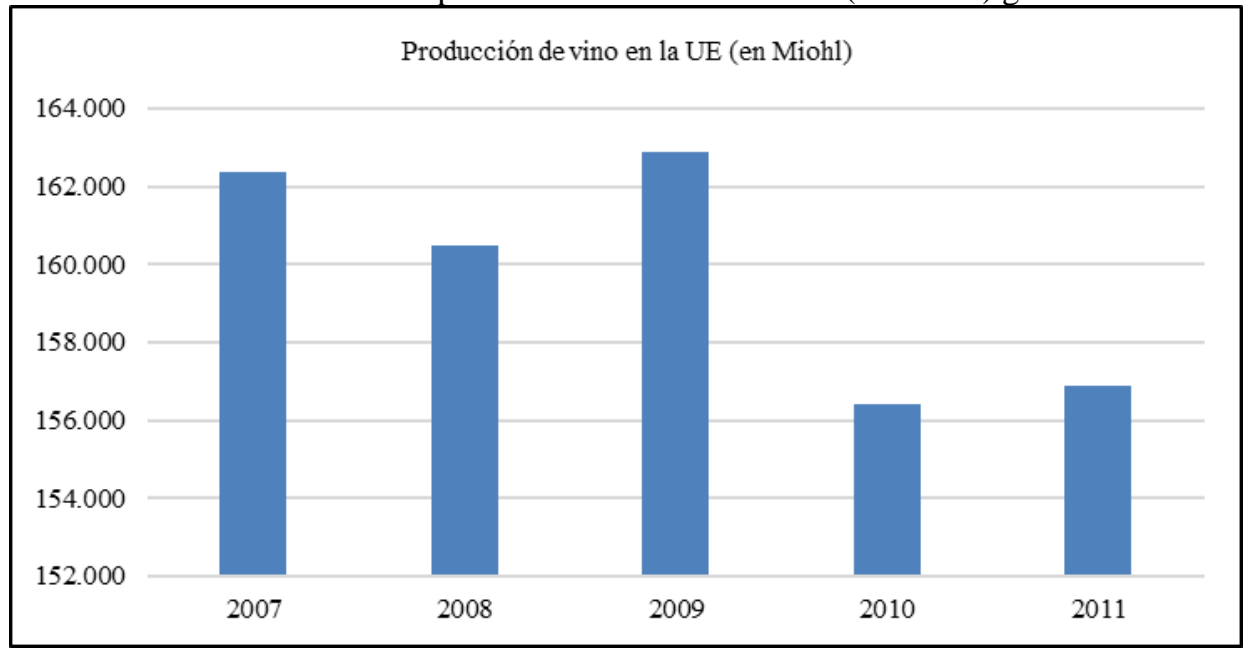

Fuente: Elaboración propia a partir de los informes del OVI (2009, 2010, 2011)

A continuación se da una pincelada de cómo ha sido la evolución en la producción de cada uno de los países de la Europa de los 27 (tabla 2).

Tabla 2

Evolución de la producción en los países de la EU de los 27

\begin{tabular}{|lrrrrrrr|}
\hline & Alemania & Austria & Grecia & España & Francia & \multicolumn{1}{c|}{ Italia } & Portugal \\
\hline $\mathbf{2 0 0 7}$ & 1026 & 2628 & 3511 & 34755 & 45672 & 45981 & 6074 \\
$\mathbf{2 0 0 8}$ & 9991 & 2993 & 3869 & 36240 & 42654 & 46970 & 5689 \\
$\mathbf{2 0 0 9}$ & 9228 & 2352 & 3366 & 35166 & 46269 & 47450 & 5868 \\
$\mathbf{2 0 1 0}$ & 6906 & 1760 & 2950 & 36093 & 45704 & 48575 & 7133 \\
\hline Promedio & 9000 & 2300 & 2450 & 35370 & 49600 & 42209 & 5925 \\
\hline Variación & 2094 & 540 & -500 & -723 & 3896 & -6366 & -1208 \\
$\mathbf{2 0 1 0} / 11$ & $(30 \%)$ & $(31 \%)$ & $(-17 \%)$ & $(-2 \%)$ & $(9 \%)$ & $(-13 \%)$ & $(-17 \%)$ \\
\hline
\end{tabular}

\begin{tabular}{|lrrrrrrrr|}
\hline & Hungría & Eslovenia & Rep. Checa & Eslovaquia & Chipre & Malta & Bulgaria & Rumania \\
\hline $\mathbf{2 0 0 7}$ & 3222 & 664 & 820 & 356 & 169 & 48 & 1796 & 5289 \\
$\mathbf{2 0 0 8}$ & 3460 & 537 & 840 & 431 & 147 & 32 & 1617 & 5159 \\
$\mathbf{2 0 0 9}$ & 3198 & 539 & 570 & 346 & 145 & 37 & 1397 & 6703 \\
$\mathbf{2 0 1 0}$ & 1966 & 545 & 564 & 130 & 117 & 39 & 1187 & 3287 \\
\hline Promedio & 2720 & 1799 & 761 & 300 & 100 & 39 & 1268 & 5400 \\
\hline Variación & 754 & 254 & 197 & 170 & -17 & 0 & 81 & 2113 \\
$\mathbf{2 0 1 0 / 1 1}$ & $(38 \%)$ & $(47 \%)$ & $(35 \%)$ & $(131 \%)$ & $(-15 \%)$ & $(0 \%)$ & $(7 \%)$ & $(64 \%)$ \\
\hline
\end{tabular}

Fuente: Elaboración propia a partir de los datos de OVI (2007, 2008, 2009, 2010, 2011) 
Si bien Francia produjo el pasado año 3,9 millones de hl más que en 2010. Italia experimenta una importante regresión de casi 7 millones de hl .Esto es debido a la política de reestructuración del viñedo. Por otro lado, Alemania y Austria aumentaron considerablemente el volumen de producción, de 9,18 a 9,6 millones de hl el país germano y de 2,3 a 2,8 millones el estado austríaco. España (debido al denominado reglamento comunitario) sufre grandes disminuciones en la producción. Por otro lado, resulta importante destacar vertiginoso aumento de Eslovaquia o Rumania durante la campaña 2010/11.

\section{Producción de vino fuera de la UE}

Fuera de la Unión Europea (tabla 3), el nivel de producción en 2011 es ligeramente superior, con 108,9 millones de hl, a 2010 (108,7 millones de hl). Hemos recogido datos de los principales productores de vino fuera de la UE 2007 a desde 2011, para posteriormente estudiar la producción en los mismos.

\section{Tabla 3}

Producción de vino de los principales países no europeos (en miles hl)

\begin{tabular}{|lccccc|}
\hline & $\mathbf{2 0 0 7}$ & $\mathbf{2 0 0 8}$ & $\mathbf{2 0 0 9}$ & $\mathbf{2 0 1 0}$ & $\mathbf{2 0 1 1}$ \\
EEUU & 19870 & 19330 & 20620 & 20700 & 18700 \\
Argentina & 15046 & 14676 & 12135 & 16300 & 15500 \\
Chile & 8227 & 8683 & 9869 & 9100 & 10600 \\
Brasil & 3502 & 3683 & 2950 & 2500 & 3500 \\
Suiza & 1040 & 1075 & 1110 & 1135 & 1210 \\
Sudáfrica & 9753 & 10165 & 9788 & 9300 & 9700 \\
Australia & 9606 & 12431 & 11600 & 11200 & 11000 \\
Nueva Zelanda & 1476 & 2052 & 2050 & 1900 & 2400 \\
\hline TOTAL & 68520 & 72095 & 70122 & 72135 & 72610 \\
\hline
\end{tabular}

Fuente: Elaboración propia a partir de los informes de OIV (2010, 2011)

EE.UU. es el país no europeo de mayor producción de vino con 18,7 millones de hl, lo que supone un descenso de más de 2 millones de hl respecto a 2010. En segundo lugar, se encuentra Argentina con 15,5 millones de hl, que disminuye sus cifras en $800.000 \mathrm{hl}$ con respecto a la producción del año anterior. En tercer puesto aparece Australia con una producción de vino de 11 millones de hl, seguida de Chile con casi un millón y medio más que en 2010.

En el caso de los EEUU en 2009 se ha registrado un ligero aumento del 6,6\% en la producción de vino respecto al 2008. Sin embargo desde 2009 se ha ido reduciendo el nivel de producción. Esto puede ser explicado a parte de por el difícil momento que atraviesa el sector vitivinícola, por el hecho de que en este país es especialmente importante la producción de uva fresca y pasa a lo que destina el mayor parte del fruto. 
Respecto a otros países de fuera de la UE, Sudáfrica pasa de producir 9,3 millones de hl en 2010 a 9,7 millones en 2011. Brasil aumenta de 2,5 millones de hl en 2010 a 3,5 millones en 2011, volviendo a registrar datos positivos tras la caída en 2010. Nueva Zelanda retorna a niveles por encima de los 2 millones de hl; tras caer en 201. Suiza, por su parte, incrementa ligeramente la producción, en $100.000 \mathrm{hl}$.

\section{Producción de vino española}

La producción de vino en España lleva siete campañas continuadas de una gran estabilidad en el entorno de los 40 millones de hectólitros (datos para vino más mosto). En la campaña 2010/2011 y según los datos del Fondo Español de Garantía Agraria (FEGA) a noviembre de 2011, la producción asciende a 40,9 millones de hectolitros, frente a los 39,3 millones de 2009/10 (dato definitivo), lo que supone un crecimiento del $4,1 \%$.

En cuanto al reparto geográfica, Castilla-La Mancha sigue siendo la principal región productora con más de la mitad de la producción total española, Extremadura sería la segunda Comunidad, seguida de Cataluña en tercer lugar (MAGRAMA, 2012).

\section{ANÁLISIS PEST PARA EL CONJUNTO DE LOS BRIC}

El contexto de crisis económica, el surgimiento del Nuevo Mundo del Vino y otros factores como el programa europeo de ayudas de arranque y los cambios de hábito de la población han posicionado a España un panorama en el que es imposible crear rentabilidad en el sector, obligándola a tener que recolocar el producto en el mercado exterior. Ante esta nueva situación en la que se encuentra el mercado del vino y concretamente sus exportaciones hay que destacar el papel destacado de los países BRIC (Brasil, Rusia, India y China). Se trata de un conjunto de países cuyos índices macro y macroeconómicos se han desviado del conjunto, siendo así emergentes mercados potenciales. Dos rasgos característicos de estos países:

a) El incremento de su Producto Interior Bruto, tanto en conjunto como de manera individualizada ha sido constante en los últimos años y con cifras muy por encima de la media de los países desarrollados.

b) Un aumento constante de su capacidad económica y, por tanto, de su capacidad de consumo. Hay una clase numerosa media-alta que forma un mercado creciente.

Como una salida a la delicada situación del sector vitivinícola es España surge así, la posibilidad de exportar a los denominados Nuevos Mercados BRIC. Éstos países engloban casi al $50 \%$ de la población mundial. Esto unido al interés y curiosidad que representan para estos ciudadanos la cultura española, y en general la mediterránea; hace que nuestro producto les sea muy atractivo. 
Su poder adquisitivo es elevado y ellos sí están dispuestos a pagar el vino español al precio que le corresponde. Es por ello que resulta muy atractivo determinar cuáles son los métodos de entrada que existen a la hora de introducirnos en esos mercados y cuál podría ser el más adecuado para cada país, siendo éste el objetivo principal de nuestro trabajo.

A la hora de poder definir una estrategia se debe conocer el entorno al que la empresa se va a enfrentar. El análisis PEST nos ofrece de manera clara y sistemática, la posibilidad de conocer, de manera genérica, algunos de los aspectos que va a influir a la hora de introducirnos en un nuevo país. Se trata del estudio de cuatro bloques temáticos: factores político legales $(\mathrm{P})$; factores económicos $(\mathrm{E})$; factores socio-culturales (S); y factores tecnológicos (T) (Bueno Campos, 1993).

Tabla 4

Aspectos de los distintos países

\begin{tabular}{|c|c|c|c|}
\hline \multicolumn{2}{|l|}{ BRASIL } & \multicolumn{2}{|l|}{ RUSIA } \\
\hline Aspectos positivos & Aspectos negativos & Aspectos positivos & Aspectos negativos \\
\hline $\begin{array}{ll}\text { Gran } & \text { mercado } \\
\text { mundial } & \end{array}$ & Gran desigualdad social & $\begin{array}{ll}\text { Potencia } & \text { mundial } \\
\text { económica } & \end{array}$ & $\begin{array}{l}\text { Población media muy } \\
\text { joven:15-55 años }\end{array}$ \\
\hline \multirow[t]{2}{*}{$\begin{array}{l}\text { Plataforma de } \\
\text { acceso a Mercosur. }\end{array}$} & $\begin{array}{ll}\text { Grandes } & \text { trabas } \\
\text { administrativas. } & \end{array}$ & $\begin{array}{l}75 \% \text { Población es } \\
\text { urbana }\end{array}$ & \\
\hline & $\begin{array}{l}30 \% \text { población menor } \\
\text { de } 30 \text { años. }\end{array}$ & $\begin{array}{l}\text { Población pudiente } \\
\text { mínima pero con } \\
\text { mucho poder. }\end{array}$ & \\
\hline \multicolumn{2}{|l|}{ INDIA } & \multicolumn{2}{|l|}{ CHINA } \\
\hline Aspectos positivos & Aspectos negativos & Aspectos positivos & Aspectos negativos \\
\hline $\begin{array}{l}\text { Segundo país en } \\
\text { tamaño. }\end{array}$ & $\begin{array}{l}\text { Renta per cápita muy } \\
\text { baja }\end{array}$ & Gran economía & $\begin{array}{l}57 \% \text { de la población es } \\
\text { rural }\end{array}$ \\
\hline \multirow[t]{2}{*}{$\begin{array}{l}\text { Acceso limitado a } \\
\text { comprar vino }\end{array}$} & $\begin{array}{l}28 \% \text { de su población es } \\
\text { urbana }\end{array}$ & $\begin{array}{l}20 \% \text { de la población } \\
\text { mundial }\end{array}$ & A penas importa vino \\
\hline & $\begin{array}{l}\text { Población media muy } \\
\text { joven, edad:14-65 años }\end{array}$ & $\begin{array}{l}\text { País más grande del } \\
\text { mundo. }\end{array}$ & $\begin{array}{l}\text { Población media muy } \\
\text { joven, edad: } 16-65 \text { años. }\end{array}$ \\
\hline
\end{tabular}

Fuente: Elaboración propia

Del análisis de cada uno de los países BRIC de forma individualizada se pueden extraer las siguientes ideas:

a) Brasil es un mercado que ofrece grandes oportunidades. La demanda de vino no es tan alta como en otros países de este grupo pero se prevés su estabilidad largo plazo. Aunque las barreras culturales, aunque existentes, se pueden salar con facilidad. Los principales obstáculo del mercado Brasileño son la compleja burocracia y la falta de infraestructuras.

b) China presenta un mercado altamente dinámico y creciente por lo que se puede presentar como una excelente oportunidad, pero los vinos españoles no están 
bien posicionados en esa zona, y necesitaríamos mucho dinero en campañas publicitarias para darnos a conocer.

c) India aparece como el país menos recomendable. En este país aunque el ritmo acelerado de crecimiento es evidente, las desigualdades en la distribución de la renta son tan altas y la cultura del vino es casi desconocida.

Tras analizar estos cuatro países se llega a la conclusión de que el país más idóneo para introducirnos es Rusia, porque es en el que los aspectos positivos superan a los negativos, por lo que vemos en él una maravillosa oportunidad para internacionalizar nuestro producto.

En conclusión parece que el mercado más adecuado es el ruso; donde nuestra cultura está occidentalizada y nuestro producto es bastante apreciado. En cuanto a la entrada a los mercados, ninguno de ellos está exento de dificultades.

La exportación está impulsada por la entrada de Rusia el 16 de diciembre de 2011 en la OMC.

En el siguiente cuadro se observa que en términos generales, Andalucía fue la sexta Comunidad Autónoma que más exportó a Rusia en 2010, siendo Cataluña la primera, tal como viene sucediendo en los últimos años. Por primera vez Andalucía llegó a mejorar su posición entre las comunidades autónomas españolas que más exportaron a Rusia; en el 2011 subió del séptimo puesto al sexto dejando atrás a Aragón y sobrepasando los 100 millones de euros.

\section{Tabla 5}

Exportaciones por comunidades autónomas españolas a Rusia.

\begin{tabular}{lccc|}
\hline & MILES DE EUROS & \% SOBRE EL TOTAL & $\% 11 / 10$ \\
Cataluña & 861.701 & $34,10 \%$ & 31,43 \\
C.Valenciana & 544.795 & $21,60 \%$ & 27,82 \\
País Vasco & 211.006 & $34,10 \%$ & 27,56 \\
Galicia & 181.621 & $7,20 \%$ & 17,17 \\
Madrid & 172.001 & $6,80 \%$ & 20,15 \\
Andalucía & 125.315 & $5,00 \%$ & 57,35 \\
Aragón & 93.342 & $3,70 \%$ & 5,29 \\
Murcia & 77.434 & $3,10 \%$ & 66,18 \\
Castilla -La Mancha & 64.037 & $2,50 \%$ & 28,11 \\
Castilla y León & 57.449 & $2,30 \%$ & $-2,05$ \\
RESTO & 137.399 & $5,40 \%$ & \\
TOTAL & 2.526 .101 & $100,00 \%$ & \\
\hline
\end{tabular}

Fuente: Elaboración propia a partir de Extenda

Como se puede apreciar en el gráfico anterior las exportaciones andaluzas han crecido entre 2010 y 2011 un 57,35\%, siendo Andalucía la segunda comunidad autónoma en la que más han crecido las exportaciones; estando por delante sólo Cataluña. Estos son unos datos bastantes alentadores de cara a futuras exportaciones con el país.

El sector agroalimentario ha sido el que tradicionalmente ha sido el principal en relación a las exportaciones de Andalucía a Rusia. Los vinos han mostrado muy buenos datos en cuanto a crecimiento (Extenda, 2012). 
Hay que tener en cuenta que los mercados a los que vamos a acceder son desconocidos para nosotros, por lo que lo más viable sería hacerlo a través de una vía que suponga menos costes y riesgos.

En cuanto a los métodos de entrada, nos parece oportuno recomendar que las primeras incursiones en estos mercados se hagan a través de la forma básica de internacionalización, es decir, la exportación teniendo un nativo que gestione el tema en el país al que nos dirigimos. Tras esta fase, estaremos en condiciones de establecer una joint-venture en el país de destino, teniendo en cuenta los costes que suponen para nosotros buscar un socio. En investigaciones posteriores se podría analizar con más detalle la estrategia de entrada en el país.

\section{CONCLUSIONES}

La necesidad de internacionalizarse en el caso de las empresas españolas, pymes en su mayoría, es evidente. El principal problema de España es que nuestro Mercado no está tan diversificado como en otros países, por lo tanto nuestro riesgo de fracaso es mayor. Por eso ahora más que nunca se necesita apoyarse en la conquista de nuevos mercados como motor de búsqueda de nuevas oportunidades.

A través de este estudio en el que se ha tenido en cuenta a nivel cuantitativo los datos estadísticos descriptivos del sector, y a nivel cualitativo de los flujos de demanda y oferta más representativos del sector, haciendo especial hincapié en los referidos a la D.O. de origen Montilla-Moriles, se ha llegado a una serie de conclusiones.

En primer lugar las cifras tanto a nivel mundial, como nacional como dentro de la D.O Montilla-Moriles que los mercados tradicionales del vino son mercados maduros y saturados. El consumo está cayendo considerablemente a lo largo de los últimos años y eso está generando un excedente que los mercados domésticos no son capaces de asumir. Por todo ello la D.O. se ha visto en la necesidad de buscar nuevos mercados más atractivos y rentables. Pero no se trata de un conjunto de países homogéneo entre sí, como hemos visto existen grandes diferencias que no los hacen igual de accesible para el vino de D.O Montilla-Moriles.

Las empresas deben realizar un análisis previo de los posibles nuevos mercados a los que se podría entrar. En nuestro caso se ha realizado un análisis PEST (político, económico, social y tecnológico) de los cuatro países que conforman el bloque BRIC y ha resultado como mercado óptimo Rusia. A pesar de ello, es importante remarcar que ninguno de los países analizados está exento de riesgos y amenazas pero Rusia es en el que éstas son mínimas para nuestro producto.

Este cambio de mercado es necesario porque debido a que los productores de vino con Denominación De Origen Montilla-Moriles tienen que competir con vinos de considerable calidad y precios muy bajos (como los de Argentina, Chile, Sudáfrica y Australia), los precios de los vinos montillanos han tenido que bajar hasta niveles muy bajos que hacen casi imposible a los productores y agricultores vivir de esta actividad. En este contexto, es necesario insistir en la idea de 
fortalecimiento del sector del vino para así obtener una imagen más unificada y un tamaño suficiente para obtener mayores ventajas en las relaciones comerciales. Por ejemplo, hay que tratar de vender el vino por sus cualidades como son la bondad para la salud o la fortaleza gastronómica del mismo y no sólo por tener una D.O. ya que con ésta cuentan numerosos vinos. En definitiva, hay que saber vender el producto.

\section{AGRADECIMIENTOS}

Me gustaría dar las gracias a las personas que dedicaron parte de su tiempo para hablar sobre proyecto y en especial, a la Cooperativa Agrícola La Unión de Montilla Sdad. Coop. Andaluza, y a las personas que me ayudan día a día en mi formación doctoral. También agradecer a la Universidad Pablo Olavide la posibilidad que me dio de llevar a cabo este proyecto en la realización de mi Trabajo de Fin de Grado en 2013, el cual me invito a seguir con la investigación.

\section{BIBLIOGRAFÍA}

AABBY, N.E y SLATER, S.F (1998), "Management influences in export performance: A review of the empirical literature 1978-88", International Marketing Review, Vol 6 (4), 7-26

ANUARIO ECONÓMICO DE CÓRDOBA (2011), Anuario Económico de Córdoba Versión Digital, Córdoba.

BUENO CAMPOS, E. (1993), Curso Básico de economía de la empresa. Un enfoque de organización, Pirámide ,Madrid.

CHEVALIER, J.M. (1995), L'Économie industrielle des Stratégies d'entrerprises, Montchrestien, Domat Économie, Paris.

DEL RÍO, M.L. y BENITO, J.L (2002), Características de formación de los decisores y comportamiento exportador de la PYME industrial alimentaria, Universidad de Santiago, Departamento de Organización de Empresas y Comercialización, Santiago.

DUNNING, J. (1998), "The eclectic paradigm of internationalization production", Journal of International Business Studies, 1-31.

EXTENDA (2012), “Memoria Extenda año 2012”. Disponible en: http://www.extenda.es/web/export/sites/extenda/archivos/comunicacion/me moria-actividades/20130626_Memoria_2012_indexada_def.pdf (accedido en 10/03/2015).

FEGA (2011), "Sector vitivinícola: producciones declaradas de vinos y mostos campaña 2010/2011". Disponible en: 
http://www.fega.es/es/PwfGcp/es/_includes/_tcmLinkFilterdotjspyiquesttcm Uriyequal28403 (accedido en 20/06/2013).

GRANT, R. M (1996), Dirección estratégica. Conceptos, técnicas y aplicaciones, Cívitas, Madrid.

GUISADO, M. (2002), Internacionalización de la empresa: estrategias de entrada en los mercados extranjeros, Pirámide, Madrid.

LA SEMANA VITIVINÍCOLA (2012), "La Semana Vitivinícola. Economía vitivinícola. Revista Digital". Disponible en: http://www.sevi.net/(accedido en 20/06/2013).

MAGRAMA (2012). "Ministerio de Agricultura, Educación y Medio Ambiente". Disponible en: http://www.magrama.gob.es/es/(accedido en 10/06/2012).

NAVAS LÓPEZ, J.E. y GUERRAS MARTÍN, L.A (2002), La dirección estratégica de la empresa. Teoría y aplicaciones, Civitas, Madrid.

OFICINA ECONÓMICA Y COMERCIAL DE LA EMBAJADA DE ESPAÑA EN MOSCÚ (2010), El mercado de vino en la Federación Rusa. Estudios de Mercado, ICEX, Madrid.

OHMAE, K. (1991), El mundo sin fronteras: poder y estrategia en la economía entrelazada, McGraw-Hill, Madrid.

PLA, J. y LEÓN, F. (2004), Dirección de empresas internacionales, Pearson, Madrid.

PORTER, M. (1988), "La competencia en las industrias globales", Información Comercial Española, 658, 71-100.

ROSSANO, E. (2001), Los determinantes del resultado exportador: Un análisis comparativo entre empresas españolas e italianas, Universidad Autónoma de Barcelona, Barcelona.

SÁINZ OCHOA, A. (2001), Análisis de los factores explicativos del éxito empresarial: una aplicación al sector de la Denominación de Origen Calificada Rioja, Universidad de La Rioja. 